\title{
Heavy Object Handling System by Cooperation of a Robot and a Crane with Multiple Wires
}

\author{
Hisashi Osumi* and Tamio Arai ${ }^{\star *}$ \\ * Department of Precision Mechanics, Faculty of Science and Engineering, \\ Chuo University, 1-13-27 Kasuga, Bunkyo-ku, Tokyo 112, JAPAN \\ ** Department of Precision Machinery Engineering, Faculty of Engineering, \\ The University of Tokyo, 7-3-1 Hongo, Bunkyo-ku, Tokyo 113, JAPAN
}

\begin{abstract}
This paper proposes a strategy for handling a heavy object by cooperation of a positioncontrolled robot and a crane with multiple wires. Installation of compliant mechanism is essential to avoid excessive inner forces caused by positioning errors of the robot and the crane. First, the necessary mechanical conditions to achieve the cooperation between position-controlled robots are reviewed. Second, compliance model of wire suspension systems are derived. After a free joint mechanism is designed assuming that the crane has three wires, a prototype of a cooperative system is developed and cooperative algorithm is proposed. The robot is position controlled as a master and the crane is velocity controlled as a slave to eliminate the displacements of free joints generated by the robot motion. The experimental results of cooperative control verified the effectiveness of the system.
\end{abstract}

\section{INTRODUCTION}

Even though heavy parts assembly is one of the most dangerous tasks in construction, it has not been automated yet. Wire suspension mechanisms are inevitable for lifting large parts such as steel beams, but their positioning accuracy is not enough for assembly because of the swinging of the parts. Thus the help of workers can not be omitted.

Authors have proposed a cooperative system between a crane and a robot instead of a human worker[1],[2]. The system can position the part with the accuracy of the installed robot and have the same payload as the crane. In this system, the assembly tasks are done as follows.

1) A part is hooked at a feeding point.

2) The part is transferred to a target point.

3) The robot catches the swinging part.

4) The robot guides the suspended part onto the assembled work.

5) The part is mated with the assembled work.

In the step 4), cooperation of the robot and the crane is needed. There have many studies proposed about cooperative control algorithms between two manipulators based on computed torque method[3],[4],[5]. But the most of algorithms can not be applied to cooperative systems between a crane and a robot, because the control performances of a manipulator and a crane are 
much different. Additionally, torque control or tension control of a huge crane is not suitable for safety. Authors proposed a simple strategy for cooperation of position-controlled robots using mechanical compliance[1],[2],[6]. Since most of popular industrial robots have position controllers, the strategy do not need to modify servo systems of robot controllers. This paper deals with the cooperative strategy based on position control.

In step 3), a prototype system for catching a swinging mass was proposed[7]. Two flexible arms were used to avoid impulsive collision forces. But it would be difficult to install the system architecture into practical site due to the complexity of the mechanisms and the control system. To realize the cooperative system in practice, crane systems should have better control performances in comparison with conventional cranes. At least, three wires are necessary for controlling all the vibration modes and also controlling both the position and the orientation of a suspended part by itself. A crane with three wires was developed and position control methods were proposed[8],[9]. Using such cranes with more than two wires, the cooperation will become much easier because the step 3), one of the most difficult steps, can be omitted.

This paper describe the design method of cooperative systems between a position controlled robot and a crane with more than two wires. First, the cooperation method is reviewed[6]. Next, wire suspension systems are modeled as springs. After describing the design method of a compliance mechanism needed for the robot, some experimental results using a cooperative system between a robot and a crane with three wires are shown.

\section{COOPERATION BETWEEN TWO POSITION CONTROLLED ROBOTS}

Cooperative system using position controllers needs mechanical compliance between robots to avoid excessive inner forces caused by mutual positioning errors of robots. The condition to avoid both the excessive inner forces due to positioning errors and the vibrations caused by the introduction of mechanical compliance is that there are linearly independent six springs between two rigid robots. The proof is as follows. Figure 1 shows a cooperative system of two robots with mechanical compliance. $\mathbf{s}_{\mathrm{i}}$ denotes the unit vector in the direction of $\mathrm{i}$-th spring and $a_{i}$ denotes the spring deflection in the direction of $\mathbf{s}_{\mathbf{i}}$.



Fig.1 Cooperative Model with Mechanical Compliance

When there are six linearly independent springs, even if the mutual positioning error e appears, it can be absorbed by six deflections of the springs calculated as Eq.(1).

$$
\mathbf{a}=S^{-1} \mathbf{e} \quad, \mathbf{a}=\left[\begin{array}{llllll}
a_{1} & a_{2} & a_{3} & a_{4} & a_{5} & a_{6}
\end{array}\right]^{T}, S=\left[\begin{array}{llllll}
\mathbf{s}_{1} & \mathbf{s}_{2} & \mathbf{s}_{3} & \mathbf{s}_{4} & \mathbf{s}_{5} & \mathbf{s}_{6}
\end{array}\right]
$$


Thus, excessive inner forces do not appear. Additionally, the deflections $a_{i}$ are determined by only geometrical constraint of $e$ and there are not any other combinations of deflections realizing $\mathbf{e}$. So, the position and orientation of the object are determined independent of force disturbance, and consequently vibrations do not occur.

Free joint mechanisms, instead of flexible mechanisms, can also be used for the cooperation by modeling the displacement of each joint as a deflection of the corresponding spring. In this case, inner force between two manipulators is always zero, thus, pushing or deforming handling objects is impossible.

\section{DERIVATION OF SPRING MODEL OF WIRE SUSPENSION SYSTEM}

When an object is suspended with $\mathrm{n}$ wires as shown in Fig.2(a), $\mathrm{n}$ geometrical constraints are generally imposed on the motion of the object. Since the object has 6 D.O.F. in 3D space, the object can move in 6-n directions according to external forces under the condition that all the wire lengths are fixed. As a result, the suspended object may vibrate in 6-n directions. Since the small vibration of the suspended object can be modeled as a second order system, the wire suspension system can be regarded as a rigid manipulator with 6-n springs at the tip as shown in Fig.2(b). The directions of these springs can be derived as follows.

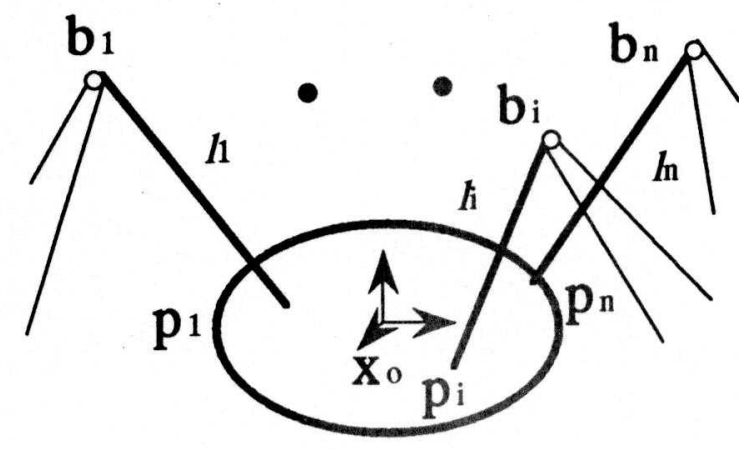

(a)

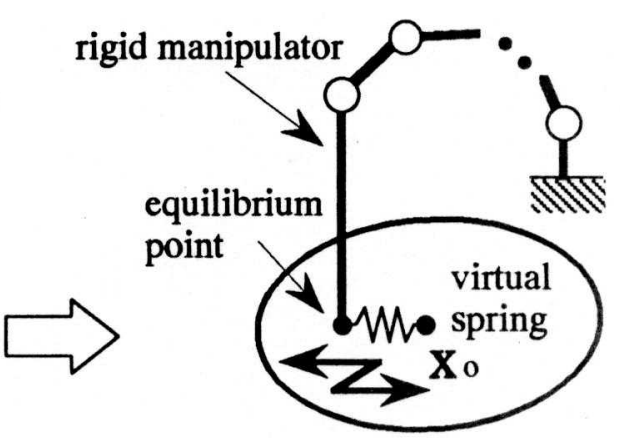

(b)

Fig.2 Model of Wire Suspension System

The geometrical constraints by wire lengths can be expressed as Eq.(2),

$f_{i}\left(\mathbf{x}_{o}\right)=\left\|\mathbf{p}_{i}\left(\mathbf{x}_{o}\right)-\mathbf{b}_{i}\right\|^{2}=l_{i}^{2}, \quad \mathbf{x}_{o}=\left[\begin{array}{llllll}x & y & z & \varphi & \boldsymbol{\theta} & \phi\end{array}\right]^{T} \quad(i=1, \cdots, n)$

where $l_{i}$ means the $i$-th wire length and $x_{0}$ means the position and the orientation vector of the object. $\mathbf{b}_{i}$ is a position vector of the tip of the crane with the $\mathrm{i}$-th wire. Assuming that all the joints of cranes and wire lengths are fixed, Eq.(3) is obtained.

$A \Delta \mathbf{x}_{o}=\mathbf{0}, \quad A=\frac{\partial \mathbf{f}}{\partial \mathbf{x}_{o}{ }^{T}}, \quad \mathbf{f}=\left[\begin{array}{lll}f_{1} & \cdots & f_{n}\end{array}\right]^{T}$

Row vectors of the coefficient matrix A of Eq. (3) express a basis of the orthogonal complimentary space of the object motion space. So, the directions of the springs of the wire 
suspended system can be obtained as a basis of the space spanned by matrix B calculated by Eq.(4),

$B=I-A^{T} A^{+T}$

where $\mathrm{I}$ is an identity matrix and $\mathrm{A}^{+\mathrm{T}}$ expresses the pseudo-inverse matrix of $\mathrm{A}^{\mathrm{T}}$.

Compliance mechanisms satisfying the condition in Chapter 2 can easily be designed considering Eq.(4). The practical design using free joints will be shown in the following chapter.

\section{DESIGN OF FREE JOINT MECHANISMS FOR COOPERATION}

\subsection{CRANE TYPE ROBOT WITH THREE WIRES}

The robot illustrated in Fig. 3 has seven degrees of freedom including three wires. Each arrow expresses a moving direction of each actuator. Each wire length can be controlled respectively. The actuator 7 is installed with the intention of increasing motion controllability in the torsion direction. Using seven actuators, the robot can position its object and reduce the residual vibrations in all directions in $3 \mathrm{D}$ space.

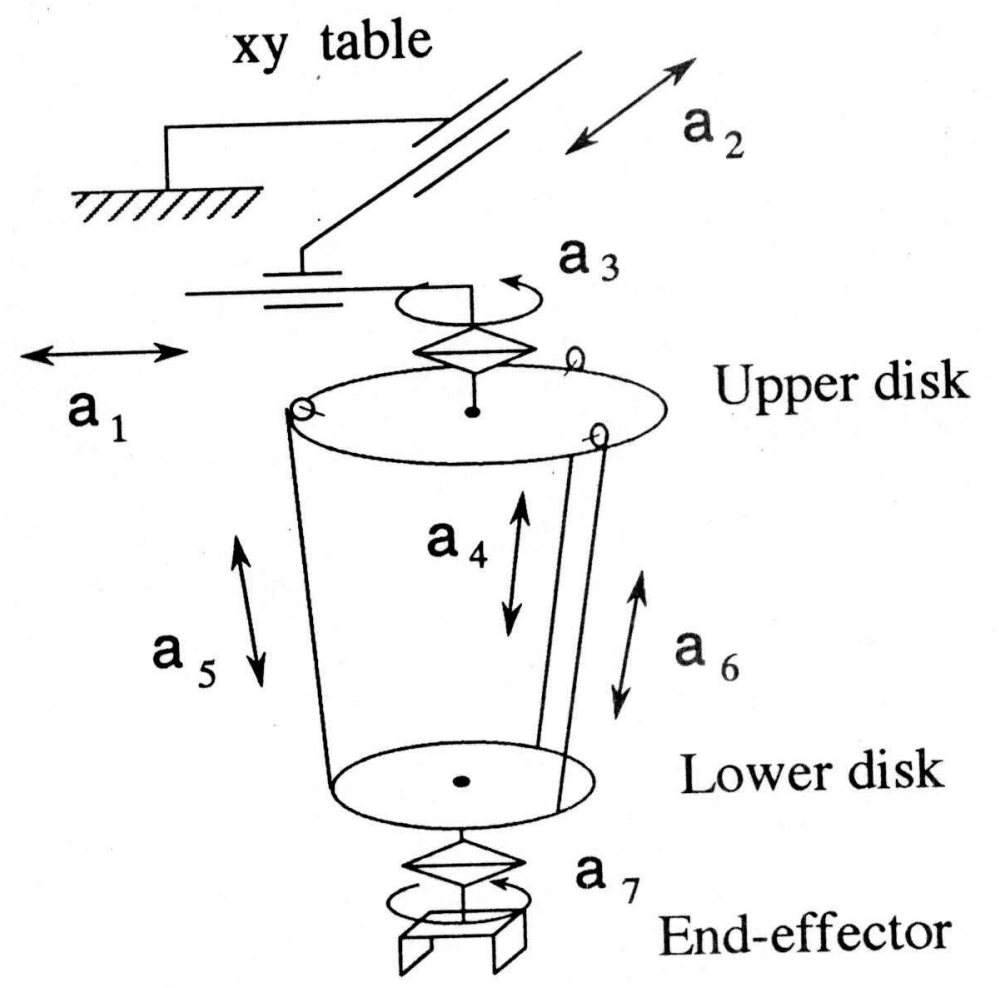

Fig. 3 Prototype of a Crane with Three Wires

Figure 4 shows the directions of vibrations of a suspended object. From the kinematic characteristics shown in Fig.4, it can be understood that this crane system can be modeled as a 7 D.O.D. rigid manipulator with three springs at the tip. The directions of the springs with respect 
to the object coordinate system $\Sigma_{\mathrm{o}}$ are as Eq.(5),

$\mathrm{s}_{c 1}=c_{1}\left[\begin{array}{llllll}0 & -d & 0 & 1 & 0 & 0\end{array}\right]^{T}$

$\mathbf{s}_{c 2}=c_{1}\left[\begin{array}{llllll}d & 0 & 0 & 0 & 1 & 0\end{array}\right]^{T}, c_{1}=1 / \sqrt{1+d^{2}}$

$\mathbf{s}_{c 3}=\left[\begin{array}{llllll}0 & 0 & 0 & 0 & 0 & 1\end{array}\right]^{T}$

where $d$ is a variable determined through kinematic parameters of the crane.
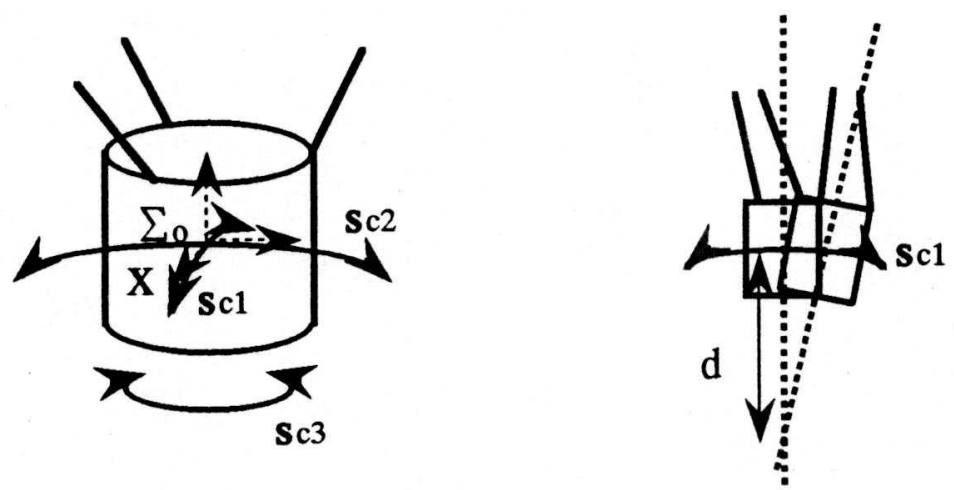

Fig.4 Vibration of a Suspended Object

So, three additional springs or free joints are needed for the cooperative robot.

\subsection{FREE JOINT MECHANISM FOR COOPERATION}

Figure 5 illustrates the designed mechanism with three free joints. The three direction vectors of installed free joints are obtained as Eq.(6).

$\mathbf{s}_{r 1}=\left[\begin{array}{llllll}0 & 0 & 0 & 1 & 0 & 0\end{array}\right]^{T}$

$\mathbf{s}_{r 2}=c_{2}\left[\begin{array}{llllll}0 & 0 & -\beta & 0 & 1 & 0\end{array}\right]^{T}, c_{2}=1 / \sqrt{1+\beta^{2}}$

$\mathrm{s}_{r 3}=\left[\begin{array}{llllll}0 & 0 & 1 & 0 & 0 & 0\end{array}\right]^{T}$

Since $\mathbf{s}_{c 1}, \mathbf{s}_{c 2}, \mathbf{s}_{c 3}$ and $\mathbf{s}_{r 1}, \mathbf{s}_{r 2}, \mathbf{s}_{r 3}$ are linearly independent of each other, it can be understood that the free joint mechanism can be applied to the cooperative system. Electro-magnetic breaks are installed in the two rotational joints and they are locked during the free motion of the robot.

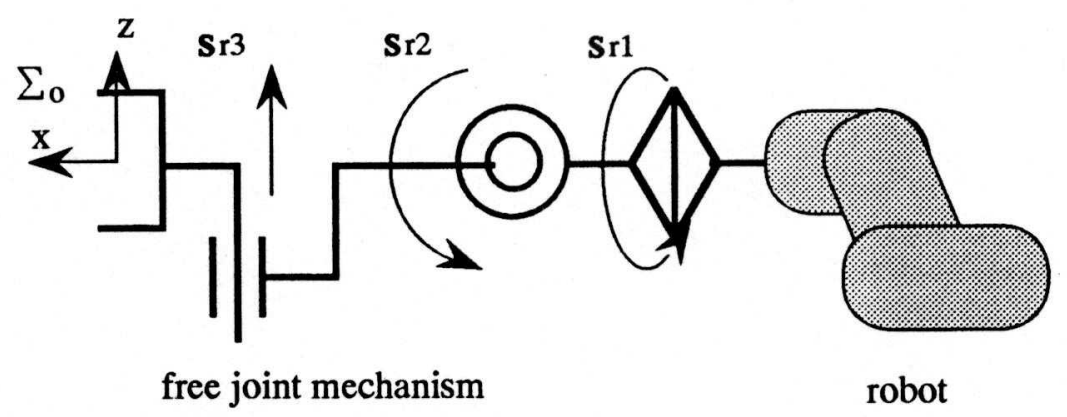

Fig. 5 Free Joint Mechanism for Cooperation 


\section{COOPERATIVE CONTROL ALGORITHM}

Figure 6 illustrates the schematic view of the cooperative system. The robot is positioncontrolled as a master, and the crane is velocity-controlled as a slave. When the robot moves, displacements of three springs of the crane and of three free joints of the robot are generated as shown in Fig.7. $a_{r 1}-a_{r 3}$ denote the displacements of the free joints and $a_{c 1}-a_{c 3}$ denote the displacements of equivalent springs of the crane. The crane is velocity controlled so as to make all the displacements zero. As a result, the object is positioned at the point where the robot stops. $a_{11}-a_{r 3}$ are measured by potentiometers and $a_{c 1}-a_{c 3}$ are measured by a gyroscope and a two axis inclinometer. Though the crane has kinematic redundancy, the actuator 7 is not used in this paper.

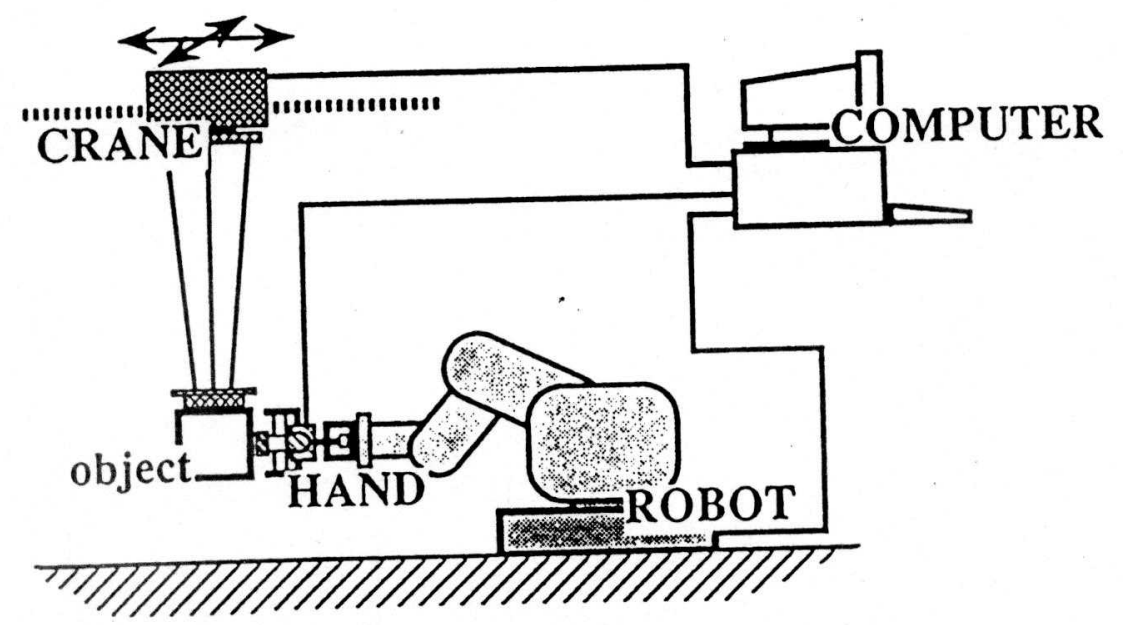

Fig. 6 Schematic View of the Cooperative System

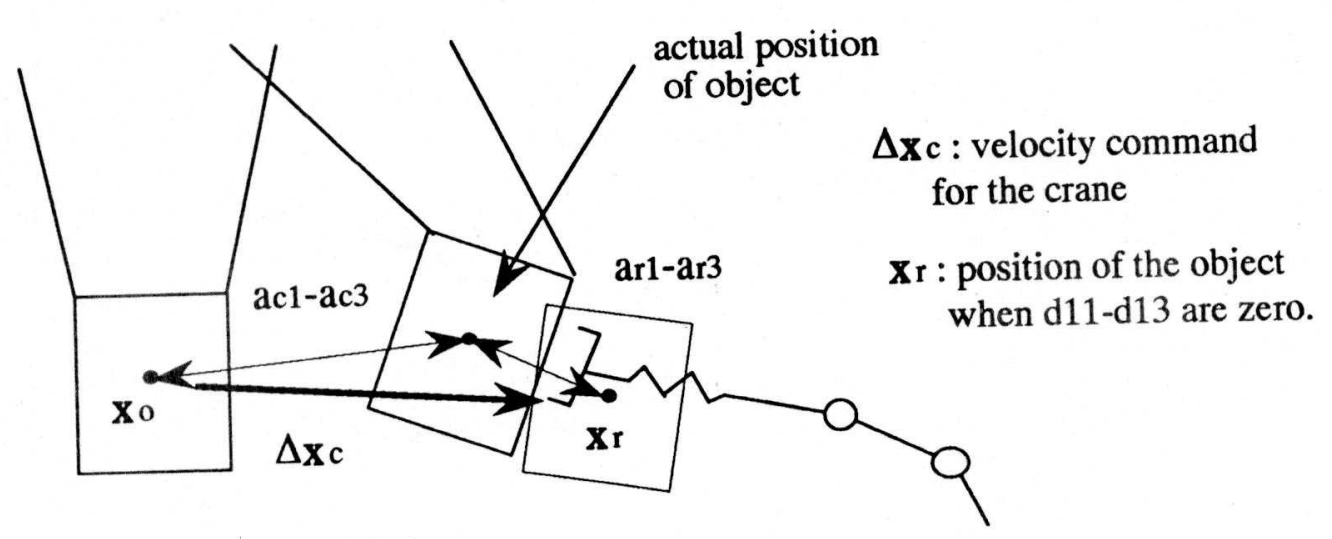

Fig.7 Relationship between Crane Motion and Displacements of Free Joints and Springs

\section{EXPERIMENT}

Figure 8 shows the configuration of the system. As a controller, PC-9801RX $(10 \mathrm{MHz}$, processor 8086,8087 ) is used. The root is MOVE MASTER RV-E2 (Mitsubishi Electronic 
Corporation) which has 6 D.O.F. and is controlled by the position commands from the computer. The working space of the crane is $1.0 \mathrm{~m}$ high, $0.9 \mathrm{~m}$ wide and $0.5 \mathrm{~m}$ deep, and the crane is velocity-controlled by the input voltages from the D/A converters of the computer. The weight of the suspended object is $15 \mathrm{Kg}$. The specifications of the sensor systems are shown in Table.1.

Table 1 Specifications of the Sensor System

\begin{tabular}{|ll|lc|}
\hline \multicolumn{2}{|c|}{ crane } & \multicolumn{1}{c|}{ robot hand } \\
\hline $\mathrm{x}, \mathrm{y}$ direction & $7.0 \times 10^{-2} \mathrm{~mm}$ & ar1 & $1.5 \times 10^{-2} \mathrm{rad}$ \\
$\mathrm{z}$ direction & $5.0 \times 10^{-2} \mathrm{~mm}$ & ar2 & $1.5 \times 10^{-2} \mathrm{rad}$ \\
rotation about $\mathrm{x}, \mathrm{y}$ axes & $5.0 \times 10^{-5} \mathrm{rad}$ & ar3 & $5.0 \times 10^{-1} \mathrm{~mm}$ \\
rotation about $\mathrm{z}$ axis & $1.7 \times 10^{-5} \mathrm{rad}$ & & \\
\hline
\end{tabular}

Assuming that the positioning of a heavy object is a desired task, the task is executed through three phases as follows.

phase 1: The object is roughly positioned by the crane. Since the crane has three wires, all the residual vibration modes can be reduced by only the crane.

phase 2: The robot catch the object. During the free motion of the robot, all breaks are locked. phase 3: After all the breaks are unlocked, the robot move to the destination slowly. The crane is feedback controlled by the signals from the inclinometer, the gyro scope, the encoders of the crane and the potentio meters of the free joints.

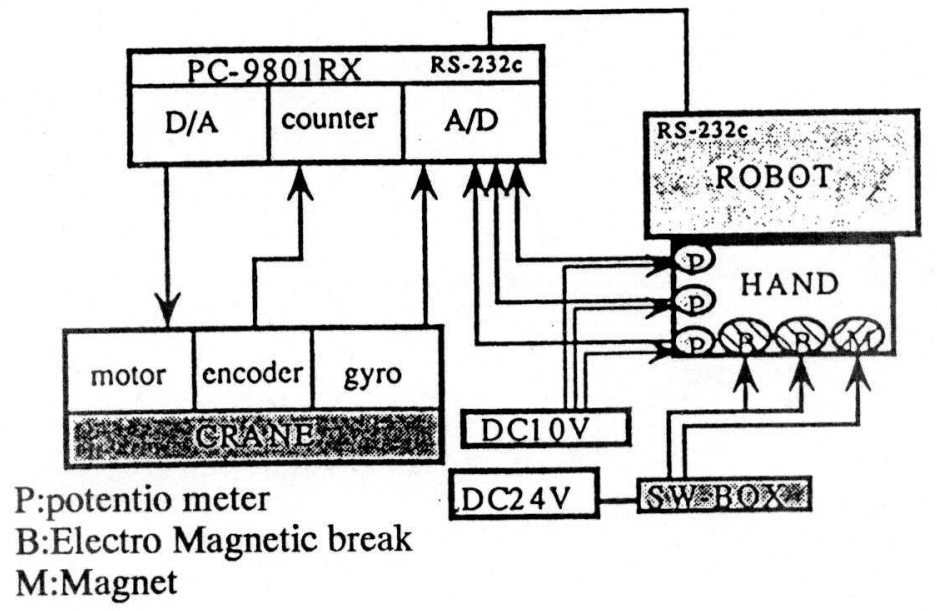

Fig. 8 Configuration of the Cooperative System

Figure 9 shows the result of the experiment of cooperation in the phase 3 . The robot moved for the first 1.7 seconds, while the crane was feedback controlled from the beginning to the end. Since the maximum speed of the crane was limited for safety, the displacements became large during the motion of the robot. But after the robot stopped, all the displacements became zero and vibration did not appear. From the result, the proposed method was verified.

\section{CONCLUSION}




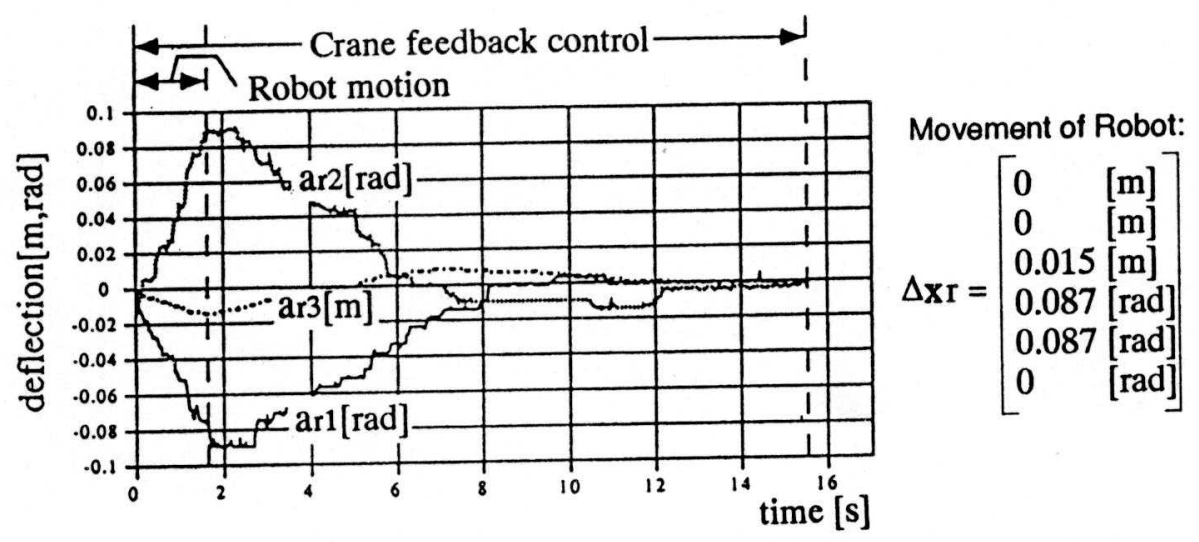

Fig.9 Displacements of Free Joints

This paper described a design method of cooperative control system between a robot and a crane with multiple wires. From the practical point of view, the system was designed based on position controller.

- The principle of the cooperative strategy for position controlled robots was reviewed.

- Wire suspension system was modeled as springs:

- The condition of mechanisms for cooperation based on position controllers was derived.

- A cooperative system between an industrial robot and a crane with three wires was developed.

- The result of an experiment of cooperative control verified the effectiveness of the system.

\section{REFERENCE}

[1] Arai, Osumi and Ohta, "Crane Control System with an Industrial Robot",Proc. 5th. Int. Symp. Robotics in Constr.,pp.747-754, 1988.

[2] Arai and Osumi, "Construction System of Heavy Parts by the Coordinated Control between a Crane and a Robot", Proc.9th Int. Symp. on Automation and Robotics in Constr.,pp.879-886, 1992.

[3] Hsu, "Coordinated Control of Multiple Manipulator Systems", IEEE Trans. on Robotics and Automation, Vo.9, No.4, pp.400-410, 1993.

[4] Xi, Tarn, and Bejczy, "Event-Based Planning and Control for Multi-Robot Coordination", Proc.1993 IEEE Int. Conf. on Robotics and Automation, pp.251-258, 1993.

[5] Kosuge, Ishikawa, Furuta and Sakai, "Control of Single-Maste Multi-Slave Manipulator System Using VIM", Proc. 1990 IEEE Int. Conf. on Robotics and Automation, pp.1172-1177, 1990.

[6] Osumi and Arai, "Cooperative System between Two Position-controlled Manipulators", Proc. IEEE Int. Conf. on Robotics and Automation, Vol.3, pp.1509-1514, 1994.

[7] Osumi, Arai and Sato, "Holding Strategy of a Suspended Object by Two Flexible Arms", J. of the Robotics Society of Japan, Vol.12, No.8, pp.1206-1213, 1994.( in Japanese )

[8] Osumi,Arai and Asama, "Development of a Crane Type Robot with Three Wires",proc.23rd Int. Symp. on Ind. Roots, pp.561-566, 1992.

[9] Osumi, Arai, Fujihira and Asama, "Crane Type Robot Suspended by Three Wires with Seven Degrees of Freedom", Proc. IFToMM-jc Int. Symp. on Theory of Machines and Mechanisms, pp.856-861, 1992. 